Rev. Saúde públ., S. Paulo

$15: 291-307,1981$

\title{
A IMPORTÂNCIA DA ASSOCIAÇÃO OBESIDADE E GRAVIDEZ *
}

\author{
Ana Cristina d'Andretta Tanaka*
}

TANAKA, A. C. d'A. A importância da associação obesidade e gravidez. Rev. Saúde públ., S. Paulo, 15:291-307, 1981.

RESUMO: Foram estudados dois grupos de gestantes, sendo um de grávidas normais e outro de obesas, $c o m$ a finalidade de reconhecer algumas características da evolução da gravidez, em mulheres obesas, e suas repercussões sobre o concepto. Foram relacionadas as seguintes variáveis: status sócio-econômico familiar, idade, altura, perímetro braquial, peso habitual, número de gestações anteriores, paridade materna, ganho de peso durante a gestação, idade gestacional, intercorrências durante a gestação, peso ao nascer e vitalidade do recém-nascido. Pelos resultados concluiu-se que as gestantes obesas são diferentes das normais e apresentam maior incidência de complicações obstétricas. Os recém-nascidos, filhos de obesas, registraram indice maior de mortalidade, principalmente no período perinatal. Houve maior incidência de prematuridade e de fetos macrossômicos, sendo a curva de distribuição de peso ao nascer diferente da dos recém-nascidos das gestantes normais. A média de peso ao nascer das crianças das gestantes obesas é maior que o das normais. Concluiu-se ainda que toda vez que a gestante obesa sofre restrição alimentar, com ganho de peso inadequado, o crescimento intra-uterino é afetado; não sendo, portanto, a época da gravidez a melhor para a obesa perder peso, mas, ao contrário, ela deveria receber uma orientação alimentar adequada. A obesidade é pois um fator de aumento do risco gravídico, que pode afetar tanto a mãe como o concepto.

UNITERMOS: Obesidade. Gestantes. Peso ao nascer. Risco gravídico.

\section{INTRODUÇAO}

Toda doença transmissivel ou não, que afeta parcela significativa da população, com repercussão sobre a saúde coletiva, se constitui um problema de saúde pública. A obesidade, portanto, apresenta-se como um problema de Saúde Pública no mundo de hoje.

Vários países desenvolvidos apresentam aumentos consideráveis na prevalência da obesidade, o que tem levado alguns deles, como por exemplo, os EUA e alguns países europeus, a considerá-la como um dos problemas mais preocupantes de Saúde Pública, na atualidade ${ }^{1,13.38}$. A prevalência da obesidade, nos EUA, na população adulta, está em torno de $25 \%$ a $45 \%{ }^{33}$.

O Brasil, apesar de ser um país em desenvolvimento, apresenta peculiarmente regiões

* Resumo da Dissertação apresentada à Faculdade de Saúde Pública da USP, para obtenção do título de Mestre, 1980, subordinada ao título "Obesidade e gravidez".

** Do Departamento de Saúde Materno-Infantil da Faculdade de Saúde Pública da USP - Av. Dr. Arnaldo, 715 - 01255 - São Paulo, SP - Brasil. 
TANAKA, A.C. d'A. A importância da associaçãr obesidade e gravidez. Rev. Saúde públ., S. Paulo, 15:291-307, 1981.

com problemas de saúde cujas caracteristicas retratam realidades sócio-econômicas diferentes. O exemplo típico deste fato é a região metropolitana de São Paulo que, pelas caracteristicas de desenvolvimento econômico, apresenta tanto doenças degenerativas e metabólicas, que são mais freqüentes em paises desenvolvidos, quanto enfermidades infecto-contagiosas e desnutrição, mais comuns nos paises em desenvolvimento.

A obesidade é uma desordem, ou doença metabólica, que se caracteriza por um aumento de peso corporal em relação à média de peso para a altura ${ }^{11,14}$, ou ainda, pode ser definida como um excesso de tecido adiposo no organismo ${ }^{1,11}$. Alguns autores consideram como pessoa obesa toda aquela que apresenta um aumento de peso de $20 \%$ em relação ao peso padrão para a altura ${ }^{6,10,13,14,32,33}$. Outros consideram que a mulher é obesa quando tiver um peso acima de $90 \mathrm{~kg} 15,20,22,24,34$.

Siqueira e col. ${ }^{30}$ elaboraram curvas para diagnóstico de estado nutricional materno e consideraram mulheres obesas aquelas que tem seu peso acima do limite superior destas.

Sendo de tiologia multi-causal, a obesidade sofre influência de fatores orgânicos, psíquicos $\mathrm{e} / \mathrm{ou}$ sociais ${ }^{17}$, que podem atuar isoladamente ou em conjunto sobre o individuo. Fatores genéticos associados com fatores ambientais, tais como maior disponibilidade de alimentos industrializados, e vida sedentária facilitam o desencadeamento da obesidade. Alguns autores afirmam que fatores sócio-econômico-culturais e ambientais exercem ação sobre a ingestão excessiva de alimentos e, com isto, provocam no indivíduo um elevado aumento de peso 1,17 . Resumindo, pode-se dizer que a obesidade resulta de um excesso relativo de ingestão calórica para o gasto energético necessário de um dado organismo levando, com isso, a um aumento na síntese de gordura ou a uma diminuição de mobilização da mesma.
Albrink ${ }^{1}$ e Robson ${ }^{25}$, entre outros autores, relatam que a obesidade é um fator de aumento de morbidade no ser humano, com maior prevalência de doenças, principalmente cardiovasculares e outras, tais como as que causam problemas respiratórios, colecistites, orteoartrites, veias varicosas, hérnias ventrais e diafragmáticas, entre outras, além do diabetes, que parece ter uma forte associaçăo com a obesidade.

Como se sabe, a gestação, por si só, leva a mulher a um risco gravídico que, quando associado a outros fatores de risco, como por exemplo, a obesidade, podem predispor tanto a gestante quanto o concepto à morbi-mortalidade materno-fetal.

Cormillot e col.1" referem autores que relatam ser a prevalência de obesidade, na mulher grávida, da ordem de 20 a $45 \%$ e que a gestação pode atuar como desencadeante da obesidade, ou como agravante desta, quando for pré-existente.

A grande maioria dos autores de trabalhos sobre o assunto afirma que a associação obesidade-gravidez aumenta a incidência de complicações maternas, levando mãe e filho a um alto risco.

A principal causa de morbidade materna entre grávidas obesas são as doenças hipertensivas, predominantemente a toxemia gravídica. Por exemplo, Petry ${ }^{2 \downarrow}$, entre outros autores, encontrou uma incidência de 35,5\% de doenças hipertensivas entre as gestantes obesas contra $14,8 \%$ no total das gestantes de seu estudo.

Em relação ao feto, as doenças hipertensivas são uma das causas de baixo peso ao nascer, e de mortalidade perinatal. É o que mostram Butler e Bonham ' na Inglaterra, Escócia e Pais de Gales, em 1968, em que $8 \%$ das mortes perinatais ocorreram em filhos de toxêmicas.

Outra complicação freqüente em obesas grávidas é a hemorragia, principalmente pós-parto $10,20,23$. 
TANAKA, A.C. d'A. A importância da associação obesidade e gravidez. Rev. Saúde públ., $\mathbf{S}$. Paulo, 15:291-307, 1981 .

No que respeita à gestação, pelo fato da mesma ser diabetogênica, o diabetes quando associado a ela apresenta uma maior incidência no grupo de gestantes obesas, como assinalam alguns autores $14,20,34$. $O$ aumento desta incidência, verificada por eles, é da ordem de $3,4 \% 20$ a $10,4 \% \%^{14,34}$.

A literatura ainda menciona outras complicações maternas, tais como pielonefrites, tromboembolismo, lesões ou infecções de cicatriz cirúrgica, que tem sua incidência aumentada em grávidas obesas $10,14,25,35$.

No período gestacional, a mulher grávida deve ganhar de 9 a $12 / \mathrm{kg}$ porém, muitas vezes, por problemas culturais, comportamentais, ambientais e/ou sociais, a gestante apresenta um ganho de peso inadequado, tanto para mais como para menos em relação aos limites recomendados.

Kawakami e col.16 verificaram que quando as gestantes ganhavam menos de $7 \mathrm{~kg}$, durante a gravidez, a média de peso dos conceptos ao nascer estava abaixo de $2.700 \mathrm{~g}$ e, se ganhassem menos de $5 \mathrm{~kg}$, esta média caia para menos de $2.500 \mathrm{~g}$. Por outro lado, quando as grávidas tinham um aumento de peso acima de $12 \mathrm{~kg}$, praticamente não havia correlação entre o ganho de peso e o peso fetal, sendo o peso do recém-nascido normal.

Springer e col. ${ }^{32}$ mostram a relação existente entre o peso pré-gestacional e o peso e a altura e circunferência craniana do concepto.

A perda ou falta de ganho de peso pode implicar em catabolismo dos tecidos maternos, provocando o desenvolvimento de cetoses, o que poderá comprometer o desenvolvimento neurológico fetal ${ }^{14}$.

Os recém-nascidos filhos de gestantes obesas, de um modo geral, são maiores que os filhos de não obesas, porém, não excessivamente maiores ${ }^{14,29,35}$. É importante notar que a incidência de baixo peso ao nascer, por retardo de crescimento intra-uterino, é menor nos filhos de mães obesas, apesar das complicações obstétricas a que este grupo está exposto. Quanto às crianças que ao nascer apresentam peso acima de $4.000 \mathrm{~g}$. encontrou-se uma incidência significativamente maior entre filhos de mães obesas do que as de não obesas ${ }^{14,35}$.

A tendência familiar da obesidade é um fator importante no tamanho do feto pois, pelo exposto, os filhos das gestantes obesas são, de um modo geral, maiores.

Este aumento de peso do recém-nascido tem sido atribuido a um aumento na adiposidade. Udall e col. ${ }^{35}$, em estudo com gestantes não diabéticas, verificaram que os filhos de gestantes obesas eram maiores que os das normais e que estes recém-nascidos tinham um aumento no tecido adiposo subcutâneo. Concluíram que o ganho de peso, durante a gestação, está associado com o aumento da obesidade e comprimento do recém-nascido, embora o elevado peso pré-gestacional se associe à obesidade do concepto, independente do comprimento deste.

Portanto, quando a gravidez desencadeia a obesidade, ou está associada a esta, leva o binômio mãe-filho a uma maior vulnerabilidade de adoecer e morrer por complicações tanto obstétricas como clínicas.

Este trabalho visa a conhecer algumas características da evolução da gravidez em mulheres obesas, bem como possiveis influências da obesidade na gravidez, que possam estar afetando o crescimento intra-uterino e a saúde e vitalidade do feto.

\section{MATERIAL E METODOS}

Para este estudo foram consideradas as gestantes inscritas no periodo de 01/07/77 a $30 / 10 / 78$ no Serviço de Pré-Natal do Centro de Saúde Geraldo de Paula Souza da Faculdade de Saúde Pública da USP (CS). Destas gestantes foram selecionadas, para um estudo prospectivo, 132, que preenchiam os seguintes critérios:

a) haviam comparecido ao Serviço de Pré- Natal para sua primeira consulta até a 25 a semana de gestação; 
TANAKA, A.C. d'A. A importáncia da associą̧ão obesidade e gravidez. Rev. Saúde públ., S. Paulo, 15:291-307, 1981.

b) não registraram nenhuma patologia própria ou associada à gravidez na primeira consulta, com exceção da obesidade;

c) não apresentaram gravidez gemelar;

d) haviam feito consulta de puerpério.

Estas gestantes foram classificadas em dois grupos sendo um de normais e outro de obesas segundo critérios de Siqueira e col.29 "curvas de ganho de peso".

Foram estudadas as variáveis: idade da gestante, nivel de status sócio-econômico familiar, antecedentes obstétricos, estatura, peso pré-gestacional, ganho de peso durante a gestaçāo, perimetro braquial materno, idade gestacional, evolução da gravidez, tipo de parto, vitalidade do recém-nascido, peso ao nascer.
Para a análise estatistica foi feito o teste do Quiquadrado $\left(x^{2}\right)$ e o teste de igualdade de duas médias estatísticas; para tal foi escolhido o método " $t$ " 31 .

O nivel de significância, tanto para o teste do $\chi^{2}$ como o de " $t$ ", foi fixado em $5 \%$.

\section{RESULTADOS E DISCUSSAO}

\subsection{Caracteristicas gerais das gestantes estudadas.}

\subsubsection{Idade das gestantes}

A média de idade da população de gestantes estudadas é de 27,66 anos; porém, as obesas apresentaram uma idade significativamente maior que as normais, como pode ser constatado na Tabela 1 .

T A B E L A 1

Idades das gestantes dos dois grupos inscritas no Centro de Saúde Geraldo de Paula Souza, no período de 1977-1978.

\begin{tabular}{l|c|c|c}
\hline Gestantes & No de casos & $\begin{array}{c}\text { Média de idade } \\
\text { (anos) }\end{array}$ & Desvio-padrão \\
\hline Normais & 81 & 26,8 & 5,6 \\
Obesas & 51 & 29,0 & 6,0 \\
\hline
\end{tabular}

Diferença significativa ao nivel de $\mathrm{p}<0,01$.

Estes dados são semelhantes aos relatados por Peckham e Christianson ${ }^{23}$, Petry ${ }^{24} \mathrm{e}$ Odell e Mengert ${ }^{22}$, o que permite concluir que, de um modo geral, as gestantes obesas apresentam idade maior que as normais.

\subsubsection{Status sócio-econômico familiar}

O nivel de status sócio-econômico familiar das gestantes que freqüentam o CS foi elaborado por Alvarenga e col. ${ }^{3}$, que analisaram a relação existente entre escolaridade da grávida e do marido, ocupação e renda do marido, possibilitando, com isto, uma estratificação em classes sociais.

O que se pode observar no estudo desta variável é que as duas populações provêm, predominantemente, das classes média e média baixa. Este fato se deve à localização geográfica do Centro de Saúde (Jardim América e Vila Madalena), o que favore um afluxo maior destas classes. Esta distribuição foi um fator limitante de análise, pois impos- 
T'ANAKA, A.C. d'A. A importancia da associação obesidade e gravidez. Rev. Saúde públ., S. Paulo, 15:291-307, 1981.

sibilitou verificar se existe alguma relação entre o status sócio-econômico familiar e o estado nutricional das gestantes, no caso específico, a obesidade.

\subsubsection{Número de gestaçōes anteriores e paridade}

Autores como Peckham e col.23 e Petry 24 relatam que as gestantes obesas apresentam número de gestações anicriores e/ou paridade maior do que as não obesas. O presente estudo constata a mesma relação, como mostram as Tabelas 2 e 3 . Apesar disto, essas diferenças, em número de gestações e paridade, não são significativas; existe, entretanto, uma tendência de as gestantes obesas terem mais gestaçōes e mais filhos que as normais.

T A B E L A 2

Número de gestações anteriores dos dois grupos de gestantes inscritas no Centro de Saúde Geraldo de Paula Souza, no periodo de 1977-1978.

\begin{tabular}{l|c|c|c}
\hline Gestantes & No de casos* & $\begin{array}{l}\text { Média de } \\
\text { gestações }\end{array}$ & Desvio-padrão \\
\hline Normais & 59 & 1,9 & 1,2 \\
Obesas & 39 & 2,4 & 1,6 \\
\hline
\end{tabular}

Diferença não significativa ao nível de $\mathbf{5} \%$.

* Excluídas as primigestas.

T A B E I A 3

Paridade dos dois grupos de gestantes inscritas no Centro de Saúde Geraldo de Paula Souza, no período de 1977-1978.

\begin{tabular}{l|cc|c}
\hline Gestantes & No de casos * & Média de paridade & Desvio-padrăo \\
\hline \multirow{2}{*}{$\begin{array}{l}\text { Normais } \\
\text { Obesas }\end{array}$} & 55 & 1,4 & 0,9 \\
\hline
\end{tabular}

Diferença não significativa ao nível de $\mathbf{5} \%$.

* Excluídas as nuliparas.

Como se pode observar nas Tabelas 1 , 2 e 3 , os grupos das gestantes obesas apresentam média de número de gestações an. teriores e de idade e paridade mais elevadas que o grupo das normais. Cabe ainda men- cionar que, nos dois grupos, o número de gestações anteriores é maior que a paridade, ocorrendo, portanto, perdas fetais anteriores da ordem de 1:4 gestações nas grávidas normais e 1:3,5 gestações para as obesas. 
TANAKA, A.C. d'A. A importância da associação obesidade e gravidez. Rev. Saúde públ., S. Paulo, 15:291-307, 1981.

\subsubsection{Estatura e peso pré-gestacional}

E interessante notar que não houve diferença na média de estatura dos dois grupos de grávidas, sendo esta de $1,56 \mathrm{~m}$.
Quanto ao peso pré-gestacional, pode-se verificar na Tabela 4 que as gestantes obesas apresentavam peso maior que as normais, sendo esta diferença estatisticamente significativa; acima de $\alpha<0,20 \%$.

T A B E L A 4

Peso pré-gestacional dos dois grupos de gestantes inscritas no Centro de Saúde Geraldo de Paula Souza, no período de 1977-1978.

\begin{tabular}{l|c|c|c}
\hline \multicolumn{1}{c|}{ Gestantes } & No de casos & $\begin{array}{c}\text { Peso habitual } \\
\text { médio }(\mathrm{kg})\end{array}$ & Desvio-padråo \\
\hline Normais & $80^{*}$ & $\mathbf{5 2 , 9}$ & $\mathbf{5 , 5}$ \\
Obesas & $49 * *$ & 66,4 & 10,8 \\
\hline
\end{tabular}

Diferenga significativa ao nível de $p<0,002$.

* 1 (um) peso pré-gestacional ignorado.

* 2 (dois) pesos pré-gestacionais ignorados.

\subsubsection{Perimetro Braquial}

A fim de se constatar se o perímetro braquial poderia servir como uma medida antropométrica para o diagnóstico de obesidade, pois devido à facilidade de sua obtenção tornou-se uma técnica viável para estudos populacionais, foi feita esta mensuraçāo nas gestantes deste estudo e constatou-se que as grávidas normais têm valores menores que as obesas. Esta diferença é estatisticamente significante ao nível de $0,20 \%$. A média do perímetro braquial para as normais foi de $27,3 \mathrm{~cm}$ e a das obesas de $32,0 \mathrm{~cm}$ com o desvio padrão de 1,9 e $2,6 \mathrm{~cm}$ respectivamente.

\subsubsection{Intercorrências durante o processo gravidico}

Vários autores mostram que ocorre uma incidência muito maior de patologias próprias e associadas ao ciclo grávido-puerperal em gestantes obesas. E também há uma maior incidência de partos distócicos neste grupo de grávidas $2,12,14,15,20,22,34$.
No presente estudo, tal fato foi observado. Porém, pelo critério rigoroso de seleção do grupo, no qual toda a gestante, quer obesa quer normal, que apresentasse patologia própria ou associada à gravidez na primeira consulta deveria ser excluida do estudo, a incidència de patologia foi pequena.

Houve apenas um caso de diabetes declarado entre as gestantes normais. Esta gestante, porém, tinha história de feto anterior macrossômico e seu peso, no decurso da gravidez, sempre esteve tangenciando o limite superior da "curva" de Siqueira e col. ${ }^{30}$. O diabetes foi diagnosticado pela prova de GTT oral. É preciso ressaltar que as gestantes obesas, mesmo com história de crianças que nasceram com peso acima de $4.000 \mathrm{~g}$, não fizeram prova de GTT oral para diagnóstico de diabetes, portanto, não fica afastada a hipótese de coexistência desta patologia nas grávidas obesas, uma vez que esta prova não foi feita sistematicamente em todas as gestantes obesas deste estudo. 
TANAKA, A.C. d'A. A importância da associaçãu obesidade e gravidez. Rev. Saúde públ., S. Paulo, 15:291-307, 1981.

A hipertensão arterial, diagnosticada na gestação, pode ser devida a toxemia ou a sindromes hipertensivas de outras causas. As gestantes obesas, que apresentaram hipertensão $(7,8 \%)$, a tiveram no último trimestre da gravidez; portanto, esta pode ser decorrente de uma sindrome toxêmica não diagnosticada.

Ocorreu também entre as grávidas obesas $3,9 \%$ de infecção urinária enquanto que as normais não apresentaram esta patologia. De um modo geral, as grávidas obesas tiveram doenças associadas e próprias da gravidez numa freqüência de $11,7 \%$ contra $2,4 \%$ das normais.

Edwards e col.14 fazem uma comparação em vários estudos com obesas $e$ as incidências das complicações obstétricas apresentadas entre eles. Com o intuito de comparar os dados deste trabalho com os de outros autores, foram associados os resultados obtidos aos da tabela de Edwards e col. ${ }^{1 \pm}$ (Tabela 5).

Verifica-se que a incidência de doenças hipertensivas, infecções urinárias e diabetes é menor em razão do exposto anteriormente. Os dados de pouco ganho de peso ou perda de peso, peso médio ao nascer e criança de tamanho excessivo se assemelham aos dos autores citados anteriormente.

A atenção ao parto é realizada em hospitais que não mantém um sistema de informação com o serviço de pré-natal do CS e, portanto, este serviço não dispõe de dados sobre as intercorrências havidas durante o período de parto e puerpério. Assim, não foi possivel saber da incidência de complicaçōes neste período, tanto no grupo das obesas como das normais. É preciso salientar a magnitude da incidência de partos operatórios (fórceps + cesárea) no presente estudo, em decorrência da indiscriminação de intervenção cirúrgica em nosso meio. A inexistência de diabetes nảo quer dizer que não ocorreu caso de diabetes nas gestantes obesas, neste estudo, mas que o diagnóstico não foi feito em razão do exposto anteriormente.

\subsubsection{Idade gestacional}

A média de idade gestacional para o total de gestantes foi de 38 semanas e meia, sendo para as normais de 39 semanas $e$ para as grávidas obesas de 38. Esta diferença não é estatisticamente significativa. A literatura menciona este mesmo fato, ou seja, não há diferenças significativas entre a idade gestacional de mulheres normais e obesas 23 É importante ressaltar que se encontrou uma incidência duas vezes maior de recém-nascidos, com merios de 37 semanas, entre as mulheres obesas. Esta foi de $8,3 \%$ nas grávidas obesas e $3,8 \%$ nas normais. A incidência de recém-nascidos com 43 semanas, e mais, foi praticamente igual nos dois grupos.

\subsubsection{Ganho de peso durante a gravidez}

O ganho de peso durante a gravidez é assunto de grande controvérsia entre obstetras, nutrólogos e outros cientistas que trabalham na área de Saúde Materna. Maham ${ }^{19}$, entre outros autores, preconiza ganho de peso de 11 a $16 \mathrm{~kg}$ para gestantes de estatura normal. Este autor ressalta, ainda, que a perda de peso durante a gravidez poderia levar a risco fetal em decorrência da queima de gordura e consequiente cetose. Nyirjesy e col. ${ }^{21}$ preconizam um ganho de peso ideal de $10 \mathrm{~kg}$ durante a gravidez. Springer e col. ${ }^{32}$ encontraram uma média de ganho de peso durante a gestação de $12 \mathrm{~kg}$, sendo que, na população de seu estudo, ocorreram casos desde ingestão alimentar livre até restrição de sódio e de calorias. Peckham e Christianson ${ }^{23}$ mostram que o grupo das grávidas obesas é o único, em seu estudo, a apresentar uma percentagem de mulheres que tiveram ganho de peso baixo, isto é, menos de $8 \mathrm{~kg}$, ou até mesmo perda de peso durante a gravidez e, portanto, foi este o grupo que teve a menor média de ganho durante a gravidez. Esses autores concluíram que, no grupo de mulheres obesas, quanto maior o peso pré-gestacional menor o ganho de peso durante 
T'ANAKA, A.C. d'A. A importancia da associação obesidade e gravidez. Rev. Saúde públ., S. Paulo, 15:291-307, 1981.

T A B E L A 5

Incidência de tipos de ocorrencias obstétricas em pacientes obesas. Comparação com outros trabalhos.

\begin{tabular}{|c|c|c|c|c|c|c|c|}
\hline $\begin{array}{l}\text { Tipos de } \\
\text { ocorrência }\end{array}$ & $\begin{array}{c}\text { Odell } \\
\& \\
\text { Mengert }\end{array}$ & Petry & $\begin{array}{l}\text { Roopnar- } \\
\text { tenesingt } \\
\text { \& Pathak }\end{array}$ & $\begin{array}{l}\text { Tracy \& } \\
\text { Miller }\end{array}$ & Freedman & Edwards & $\begin{array}{c}\text { Presente } \\
\text { estudo }\end{array}$ \\
\hline $\begin{array}{l}\text { Doenças } \\
\text { Hipertensivas }\end{array}$ & 31,4 & 35,5 & 41,0 & 41,7 & 66,0 & 22,6 & 7,8 \\
\hline $\begin{array}{l}\text { Pouco ganho e/ou } \\
\text { perda de peso }\end{array}$ & - & - & - & - & - & 31,2 & 23,5 \\
\hline $\begin{array}{l}\text { Diabetes (de clarado } \\
\text { e gestacional) }\end{array}$ & - & - & 40,0 & 10,4 & 17,9 & 12,5 & - \\
\hline $\begin{array}{l}\text { Hemorragia } \\
\text { Pós-parto }\end{array}$ & 7,1 & 0,8 & 15,0 & 2,0 & - & 20,1 & \\
\hline $\begin{array}{l}\text { Infecçðes } \\
\text { Urinárias }\end{array}$ & - & - & 5,0 & 10,4 & - & 10,1 & 3,9 \\
\hline $\begin{array}{l}\text { Apresentação } \\
\text { Pélvica }\end{array}$ & 5,5 & 4,6 & - & 8,3 & 19,9 & 2,4 & \\
\hline Cesãrea & 1,2 & 1,5 & 17,0 & 6,2 & 14,3 & 11,0 & 48,0 \\
\hline $\begin{array}{l}\text { Parto. Operatorio } \\
(\mathrm{F}+\mathrm{C})\end{array}$ & 11,0 & 31.1 & 19,0 & 35,4 & - & 21.0 & 51,0 \\
\hline $\begin{array}{l}\text { Peso médio } \\
\text { ao nascer }(\mathrm{Kg})\end{array}$ & 3,580 & 3,807 & - & 4,025 & 3,532 & 3,459 & 3,359 \\
\hline $\begin{array}{l}\text { Baixo peso } \\
\text { ao nascer }\end{array}$ & 3,6 & 2,7 & 8,0 & - & - & 4,8 & 10,4 \\
\hline $\begin{array}{l}\text { Crianças de } \\
\text { tamanho excessivo }\end{array}$ & 22,6 & 36,7 & 16,0 & - & - & 20,6 & 18,8 \\
\hline
\end{tabular}

Extraída de Edwards, z.E. e col.14, com acréscimo dos dados obtidos no presente trabalho.

a gravidez e maior o peso de recém-nascido.

No presente estudo constatou-se que o ganho de peso das gestantes estudadas oscilou entre o mínimo de $-0,5 \mathrm{~kg}$ e o máximo de $19,5 \mathrm{~kg}$, sendo que a média de ganho de peso foi de $9,2 \mathrm{~kg}$. A Tabela 6 mostra a média de ganho de peso para as gestantes normais e para as obesas. Como se pode notar, as grávidas normais têm ganho de peso médio maior que as obesas, apesar desta diferença não ser estatisticamente significante. Isto possivelmente se deve à influência đa orientação alimentar dos obstetras sobre as gestantes obesas, pois recomendam pequeno ganho de peso a este grupo.

A incidência de baixo ganho de peso (8kg), ou perda de peso, observado no presente estudo, foi de $41 \%$ entre as gestantes obesas enquanto que, entre as gestantes normais, foi de $27,7 \%$. 
TANAKA, A.C. d'A. A importância da associação obesidade e gravidez. Rev. Saúde públ., S. Paulo, 15:291-307, 1981.

Vale a pena ressaltar que nenhuma ges- até perda de peso $(-0,5 \mathrm{~kg})$ durante o tante normal teve ganho de peso abaixo de periodo gravídico. $2,7 \mathrm{~kg}$ enquanto que, entre as obesas, houve

T A B E L A 6

Ganho de peso durante a gestação dos grupos de gestantes inscritas no Centro de Saúde Geraldo de Paula Souza, no período de 1977-1978.

\begin{tabular}{l|c|c|c}
\hline \multicolumn{1}{c|}{ Gestantes } & No de casos & $\begin{array}{c}\text { Ganho de peso } \\
(\mathrm{Kg})\end{array}$ & Destio-padrão \\
\hline Normais & $80 *$ & 9,4 & 3,8 \\
Obesas & $50 *$ & 9,0 & 4,3 \\
\hline
\end{tabular}

Não há diferença significativa a nível de $5 \%$.

* 1 caso de cada grupo com apenas 1 consulta de pré-natal.

\subsubsection{Tipo de parto}

Em nosso meio, a cesárea é tão indiscriminada que torna difícil verificar a real incidência desta, relacionada a problemas obstétricos propriamente ditos. Como consta na Tabela 7 , a incidência de cesárea é alta para os dois grupos.

\section{T A B E I A 7}

Tipo de parto nos dois grupos de gestantes inscritas no Centro de Saúde Geraldo ce Paula So:za, no período de 1977-1978.

\begin{tabular}{l|cc|c|c}
\hline & Gestantes & \multicolumn{2}{|c}{ Obesas } \\
\hline $\begin{array}{l}\text { Tipo } \\
\text { de parto }\end{array}$ & Normais & No de casos & $\%$ \\
\hline Normal & 45 & 55,6 & 25 & 49,0 \\
Fórceps & 8 & 9,8 & 3 & 5,9 \\
Cesárea & 28 & 34,6 & 23 & 45,1 \\
\hline Total & 81 & 100,0 & 51 & 100,0 \\
\hline$x^{2}$ ob $=1,75$ & $x^{2}$ critico $=5,99$.
\end{tabular}

Diferença não significativa ao nivel de $5 \%$.

Fisher e Frey ${ }^{15}$, em seu estudo, mostram apenas $1 \%$ de cesáreas em gestantes obesas contra $2 \%$ nas normais. Odell e Mengert 22 em estudo realizado apenas com gestantes obesas constataram uma incidência de partos operatórios (Fórceps + Cesárea) de $11 \%$;
Petry ${ }^{24}$, Matthews e Bruck 20 observaram apenas $1,5 \%$ de cesáreas em mulheres obesas. De um modo geral, a obesidade não determina aumento de incidência de cesáreas, apesar de os recém-nascidos serem 
MANAKA, A.C. d'A. A importância da associação obesidade e graridez. Rer. Saúde pübl., S. Paulo, 15:291-307, 1981.

maiores e de haver maior incidência de complicações obstétricas neste grupo.

Portanto, não se justifica a alta incidència de cesárea nas grávidas obesas $(45,1 \%)$ e muito menos nas normais $(34,6 \%)$. Assim sendo, não há como poder avaliar a diferença encontrada, pois a indicação de cesáreas nos dois grupos não decorreu provavelmente de conduta obstétrica adequada.

\subsection{Estudo do recém-nascido}

\section{2 .1 obitos fetais e neonatais}

A literatura sobre obesidade e gravidez pouco se refere a óbitos fetais e neonatais. Apenas se encontram menções em Matthews e Bruck ${ }^{20}$ (1938) e Odell e Mengert 22 (1942), que foram os primeiros a estudar o assunto. Para os primeiros, a incidência de óbito fetal e neonatal foi de $12,3 \%$ e, no segundo estudo, de $4,2 \%$; ambos os casos foram óbitos fetais intermediários, (20 a 27 semanas de gestação), tardios (28 semanas de gestação e mais) e neonatais (de 0 a 28 dias de vida). Neste presente estudo a incidência de óbitos fetais (intermediárias, tardias) e neonatais, em mulheres obesas, foi de $17,7 \%$, enquanto que entre mulheres normais foi de $4,9 \%$. Portanto, a incidência foi 3,5 vezes maior no grupo de mulheres obesas, o que parece ser elevado.

Esta diferença foi estatisticamente significativa, o que permite afirmar que o risco de óbito fetal é maior no grupo das obesas. A comparação entre os dados da literatura 20,22 com os deste estudo mostra que a incidência de perdas fetais e neonatais, em gestantes obesas, é ainda maior, o que mostra que apesar dos avanços tecnológicos, outros fatores estão exercendo influência sobre esta incidência.

\subsubsection{Mortalidade perinatal}

Pela incidência de perdas fetais e neonatais no grupo de mulheres obesas parece importante estudar o coeficiente de mortalidade perinatal. Esta taxa é, para o total de gestantes, de $47,6 \%$ nascimentos. Ao se desdobrar nos dois grupos ela passa a $12,7 \%$ nascimentos para as mulheres normais e 106,4\% nascimentos para as obesas, - que indica que a obesidade pode estar influenciando, direta ou indiretamente, este coeficiente, mostrando, assim, vulnerabilidade maior a que este grupo está exposto.

Como se sabe, o periodo perinatal é aquele entre a $28^{\text {a }}$ semana de gestação até os sete dias de vida extra-uterina; logo, influências, quer biológicas quer ambientais, atuando sobre a gestante, irão ter repercussões sobre o binômio mãe-filho.

Ao se comparar estes resultados com o de outros paises, pode-se verificar que este coeficiente está muito elevado. No Reino Unido, em $1970^{\circ}$, era da ordem de $22 \%$, e em Cuba $(1973)^{4}$ de $24,6 \%$ nascimentos. O dado deste estudo aproxima-se ao de Portugal, em 1966, que era de $43,6^{\circ} /$ oo nascimentos, e é bem superior ao da Suécia que, nesta mesma época, era de $18,9 \%$ nascimentos 27 . Comparando o coeficiente de mortalidade perinatal do grupo de gestantes obesas $(106,4 \%$ nascimentos) com o mesmo coeficiente para São Paulo $(42,04 \%$ nascidos vivos 18) apesar deste ter no seu denominador apenas nascidos vivos, verifica-se que ele é mais de duas vezes maior que o coeficiente de mortalidade perinatal para o município de São Paulo. Isso mostra que ele está muito alto principalmente se levar em conta que estas gestantes são predominantemente de classe média e, portanto, deveriam ter um coeficiente mais baixo que o da Capital de São Paulo como um todo. Outro fato importante a relatar é que a incidência de mortalidade perinatal, no grupo de obesas do estudo de Petry ${ }^{24}$, foi de $3,7 \%$, inferior ao encontrado neste estudo $(10,6 \%)$. Portanto, a gestante obesa se expõe a risco muito maior de perdas no periodo perinatal, apesar de receber o mesmo tipo de assistência, quer pré-natal como de parto, o que permite concluir, com 
TANAKA, A.C. d’A. A importância da associação obesidade e gravidez. Rev. Saúde pübl., s. Paulo, 15:291-307, 1981.

base, neste estudo, que a obesidade é um fator de risco, podendo afetar o concepto, ou seja, a obesidade acaba sendo também um risco fetal além de materno.

\subsubsection{Peso ao nascer}

É esta, sem dúvida, a variável mais crítica do estudo e a mais estudada por diversos autores, pois ela parece refletir, direta- mente, os agravos à saúde da mãe, ocorridos durante o periodo pré-gestacional, gestacional e intergestacional.

De um modo geral, os recém-nascidos filhos de mulheres normais têm seu peso médio, ao nascer, menor que os das obesas $14,23,24,33$. Esta diferença também foi observada no presente estudo (Tabela 8); porém não é estatisticamente significativa.

T A B E L A 8

Média de peso ao nascer dos recém-nascidos nos dois grupos de gestantes inscritas no Centro de Saúde Geraldo de Paula Souza, no periodo de 1977-1978.

\begin{tabular}{l|c|cc}
\hline Gestantes & No de casos & Média de peso (g) & Desvio-padrảo \\
\hline Normais & $80 *$ & $3.245,9$ & 552,0 \\
Obesas & $48 * *$ & $3.359,4$ & 764,7 \\
\hline
\end{tabular}

Diferenças não significativas ao nível de $\mathbf{5 \%}$

* 1 Perda fetal com peso ignorado.

* 3 Perdas fetaís com peso Ignorado.

A partir deste momento, toda vez que se trabalhar com peso do recém-nascido, ter-se-á 128 gestantes, sendo 80 normais e 48 obesas por terem sido registradas 4 perdas fetais com peso ignorado.

E interessante notar que a distribuição de peso ao nascer é diferente entre os recém-nascidos de mulheres normais $e$ obesas. A Fig. ilustra esta diferença. Tompkins e Wiell ${ }^{33}$, encontram em seu trabalho uma distribuição bem semelhante à deste estudo.

Como a Fig. mostra, as mulheres normais têm $3,8 \%$ de crianças com menos de $2.500 \mathrm{~g}$ enquanto que as obesas $10,4 \%$. Quanto às crianças de $4.000 \mathrm{~g}$ ou mais, as percentagens foram de $5 \%$ e $18,8 \%$ nos grupos das normais e obesas respectivamente. Além de haver crianças com peso inadequado, recém-nascidos pequenos $<2.500 \mathrm{~g}$ e grandes $>4.000 \mathrm{~g}$, sendo $8,8 \%$

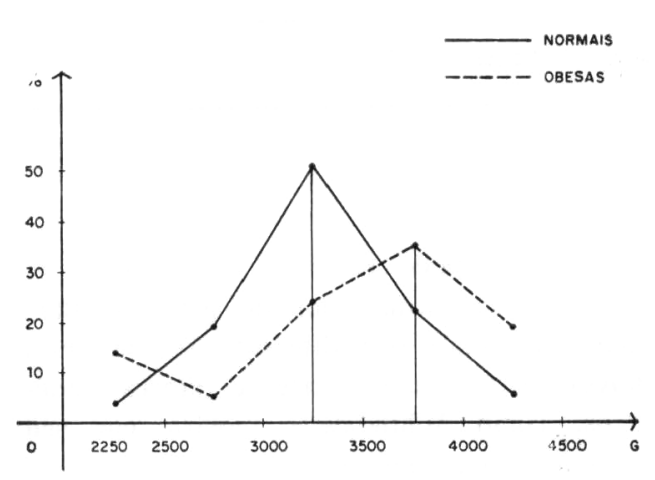

Fig. - Distribuição de peso ao nascer, dos recémnascidos nos dois grupos de gestantes em estudo.

nas gestantes normais contra $29,2 \%$ nas obesas, também há uma inversão, ou seja, $51 \%$ de crianças com $3.000 \mathrm{~g}$ a $3.500 \mathrm{~g}$ e $22 \%$ de 3.500 a $4.000 \mathrm{~g}$ nas normais e $29 \%$ e $35 \%$ nas obesas, respectivamente. As 
T'ANAKA, A.C. d'A. A importancia da associação obesidade e gravidez. Rev. Saúde públ., S. Paulo, 15:291-307, 1981.

gestantes obesas tiveram poucas crianças com peso entre 2.500 a $3.000 \mathrm{~g}$. A Tabela 9 evidencia esta distribuição de peso ao nascer nos dois grupos de gestantes. Com isto, verifica-se que um entre 3 filhos de obesas estão nascendo com peso inadequado, ou com peso inferior a $2.500 \mathrm{~g}$ ou superior a $4.000 \mathrm{~g}$.
Batista ${ }^{5}$, mostra uma incidência elevada de morbidade neonatal em fetos macrossômicos não relacionada ao diabetes materno, mas a problemas de tocotraumatismo, hipocalcemia a malformações indicando, com isto, que fetos grandes apresentam risco de adoecer muito maior que os normais.

T A B E L A 9

Distribuição de peso dos recém-nascidos dos dois grupos de gestantes inscritas no Centro de Saúde Geraldo de Paula Souza, no período de 1977-1978.

\begin{tabular}{|c|c|c|c|c|}
\hline \multirow[b]{2}{*}{ Peso (g) } & \multicolumn{2}{|c|}{ Normal } & \multicolumn{2}{|c|}{ Obesas } \\
\hline & No & $\%$ & Ne & $\%$ \\
\hline$<2.500$ & 3 & 3,8 & 5 & 10,4 \\
\hline $2.500-3.000$ & 14 & 17,5 & 3 & 6,3 \\
\hline $3.000 \mid-3.500$ & 41 & $\mathbf{5 1 , 2}$ & 14 & 29,2 \\
\hline $3.500 \vdash 4.000$ & 18 & 22.5 & 17 & 35,4 \\
\hline $4.000 \mathrm{e}+$ & 4 & $\mathbf{5 , 0}$ & 9 & 18,8 \\
\hline Total & 80 & 100,0 & 48 & 100,0 \\
\hline
\end{tabular}

Como se pode notar, a distribuição de peso dos recém-nascidos de grávidas normais e obesas é bem diferente. Isto se deve, talvez, em pequena parte a fatores genéticos, como Whitelaw ${ }^{37}$ mostra em seu trabalho; mães obesas geram filhos com um aumento de gordura subcutânea, portanto, mais pesados; mas deve-se principalmente a fatores ambientais e comportamentais, que possam estar exercendo influência sobre as gestantes obesas. E preciso lembrar, mais uma vez, que talvez o ganho de peso, durante a gravidez, nas obesas, que é por vezes restringido, possa estar influindo esta distribuição.
Para descartar a possibilidade da variável paridade estar interferindo na distribuição de peso foi estabelecida uma possivel relação entre as duas variveis e pôde-se constatar que não houve de fato relação entre elas; logo, a variável paridade provavelmente não está exercendo influência na distribuição do peso ao nascer.

Outra variável, que poderia estar influindo nesta distribuição, é a referente a complicações obstétricas. Porém, a única associação encontrada diz respeito ao peso ao nascer, de filho de mãe diabética; as demais não tiveram associação estatisticamente significativa com o peso ao nascer. 
I'ANAKA, A.C. d'A. A importancia da associação obesidade e gravidez. Rev. Saúde públ., S. Paulo, 15:291-307, 1981.

\subsubsection{Relação entre peso ao nascer $e$ variáveis maternas.}

\subsubsection{1 ldade, estatura e perimetro braquial materno}

As variáveis idade, estatura, e perímetro braquial materno parecem não estar influindo na distribuição de peso ao nascer, dos grupos em estudo. Até mesmo a literatura é pobre em relação a estas variáveis e apenas alguns autores mencionaram esta relação; Castilho e col.8, mostram não ter havido associação entre 0 peso do recém-nascido, idade e estatura da mãe, análise esta feita pela regressão múltipla do tipo "stepwise".

\subsubsection{Peso ao nascer nas gestaçöes anteriores}

Parece haver uma relação positiva entre o peso do recém-nascido da gestação anterior e o da gestação em estudo, pois eles parecem refletir uma história familiar. Mães que tiveram filhos com peso inadequado têm filhos com peso inadequado, e as que tiveram filhos com peso adequado geram filhos com pesos adequados. Isto ocorreu nos dois grupos de gestantes de um modo geral. Assim, das 5 gestantes obesas, que tiveram fetos acima de $4.000 \mathrm{~g}, 3$ tiveram filhos anteriores com $4.000 \mathrm{~g}$ on mais $\mathrm{e}$ duas com 3.500 a $4.000 \mathrm{~g}$.

Entre as normais, das 3 que tiveram filhos acima de $4.000 \mathrm{~g}$, uma teve um filho anterior com $4.000 \mathrm{~g}$ e mais, e duas tiveram filhos entre 3.000 e $3.500 \mathrm{~g}$.

Todas as obesas que tiveram filhos de $2.500 \mathrm{~g}$ e menos, tinham outros filhos anteriores com pesos entre 2.500 e $3.000 \mathrm{~g}$. Quanto às normais, das 3 que tiveram filhos de $2.500 \mathrm{~g}$ e menos, uma teve também um filho de $2.500 \mathrm{~g}$ e menos; outra entre 2.500 e $3.000 \mathrm{~g}$ e a última entre 3.500 e $4.000 \mathrm{~g}$. Rush e col.26, em estudo feito no Harlem, New York, encontraram também esta associação entre história de filhos de baixo peso, com baixo peso ao nascer, nas crianças da gestação atual. Nesse estudo, é a variável (baixo peso ao nascer anterior) que tem a associação mais forte com o peso do recém-nascido atual.

Portanto, além dos fatores ambientais (nutrição), parece existir componente genético que também influi no peso do feto ao nascer.

\subsubsection{Idade gestacional}

A relação entre esta variável e o peso do recém-nascido mostrou que quase todas as crianças de menos de 37 semanas $(5,3 \%)$ apresentam peso inferior a $2.500 \mathrm{~g}$ e as de 37 a 44 semanas distribuem-se entre os pesos de $2.500 \mathrm{~g}$ a $4.000 \mathrm{~g}$ ou mais. Há uma excesão: um caso de recém-nascido de gestante obesa, com 41 semanas e pesando $2.420 \mathrm{~g}$; porém, era uma criança portadora de anomalia congênita grave.

A incidência de prematuridade nas gestantes obesas foi de $8,3 \%$ enquanto que nas normais foi de $3,8 \%$, mostrando que as obesas apresentam uma incidência duas vezes maior de prematuridade que as grávidas normais.

Pelo que se pode observar, o baixo peso que se encontrou neste estudo, entre as gestantes obesas, se deve à prematuridade e não a retardo de crescimento intra-uterino. Isto difere de gestantes normais e principalmente de desnutridas, como observa Siqueira ${ }^{29}$ que 0 baixo peso deste último grupo se deve principalmente ao retardo de crescimento intra-uterino, ao PIG (pequeno para idade gestacional) e não aos prematuros.

\subsubsection{Peso pré-gestacional}

Esta variável parece ser a que pode trazer algumas explicações sobre o comportamento do peso do recém-nascido. Teriam os recém-nascidos, filhos de mulheres com maior peso pré-gestacional, um peso mais alto ao nascer? 
TANAKA, A.C. d'A. A importancia da associaçãc obesidade e gravidez. Rev. Saúde públ., S. Paulo, 15:291-307, 1981.

Esta indagação parece ser verdadeira. Peckham e Christianson ${ }^{24}$, Simpson e col. ${ }^{28}$, Weiss e Jackson ${ }^{36}$ e Rush ${ }^{26}$ entre outros relatam uma associação positiva entre o peso pré-gestacional e o peso do concepto ao nascer: quanto maior o peso pré-gestacional maior o peso do recém-nascido.

Esta relação também está presente neste estudo, ao se verificar que a média de peso pré-gestacional das gestantes obesas é maior que as das normais (Tabela 4), sendo a média de peso do recém-nascido maior nas obesas que nas normais (Tabela 8). Ao detalhar o peso ao nascer pelo peso pré-gestacional, pode-se observar que mutheres com baixo peso pré-gestacional geram filhos menores (Tabela 10 ).

TABELA 10

Relação entre peso pré-gestacional e peso ao nascer dos dois grupos de gestantes inscritas no Centro de Saúde Geraldo de Paula Souza, no periodo de 1977-1978.

\begin{tabular}{|c|c|c|}
\hline Peso $\begin{array}{c}\text { Média de } \\
\text { pré-gestacional }(\mathrm{Kg})\end{array}$ & Nu de casos * & $\begin{array}{l}\text { Médía de peso } \\
\text { ao nascer } \\
\text { (g) }\end{array}$ \\
\hline$<\mathbf{5 0}$ & 20 & $3.245,5(1)$ \\
\hline $50-60$ & 57 & $3.337,9(2)$ \\
\hline $60 !-70$ & 30 & 3.528 .0 (3) \\
\hline $70 \mathrm{e}+$ & 11 & $3.624,6(4)$ \\
\hline
\end{tabular}

Diferença significativa ao nivel de $p<0.05$ entre as médias de peso ao nascer dos ftens 1 e 3,1 e 4 .

* 11 casos com menos de 37 semanas e, 3 casos sem peso pré-gestacional.

A Tabela 10 mostra que o ganho de peso semanal, quando agrupados os dois grupos (gestantes normais e obesas), e separados por faixas de peso pré-gestacional não tem interferência sobre 0 peso do recém-nascido. Isso porque os extremos dos ganhos de peso excessivo, ou perda e/ou pouco ganho, estão afetando diretamente esta média. Cabe, ainda, ressaltar que, no grupo das obesas, as que tiveram um ganho de peso, durante a gravidez, inferior a $8 \mathrm{~kg}$, geram filhos menores $(3.242 \mathrm{~g})$ que as que ganharam mais de $8 \mathrm{~kg}$. Nestas últimas, os recém-nascidos tiveram uma média de peso de $3.656 \mathrm{~g}$, o que mostra que, quando o ganho de peso, durante a gestação é inadequado, o recém-nascido tem um retardo no seu crescimento intra-uterino. Portanto, uma vez mais ressalta-se que: não é o periodo de gravidez o momento mais propício para a gestante obesa perder ou dejxar de ganhar peso.

Um dos fatores de maior importância a um adequado crescimento e desenvolvimento intra-uterino do concepto é, portanto, o estado nutricional em que se encontra a mulher, durante e depois do ciclo gravidico.

\subsubsection{Ganho de peso semanal}

O ganho de peso durante a gestação e de grande importância sobre o peso do recém-nascido. Como se sabe, a perda 
TANAKA, A.C. d'A. A importancia da associaçăo obesidade e gravidez. Rev. Saúde públ., $\mathbf{S}$. Paulo, 15:291-307, 1981.

deste, ou mesmo o pouco ganho de peso durante a gravidez, faz com que o feto tenha um retardo em seu crescimento intra-uterino e, portanto, pode apresentar comprometimento, tanto a nivel pondero-estatural como em número de células, conforme o periodo gestacional em que $o$ feto foi atingido. Vários estudos mostram a cor- relação entre ganho de peso durante a gestação e o peso do concepto ao nascer $21,23,29$. Pode-se observar esta relação no presente estudo e verificar, também, que existe uma diferença significativa entre os pesos dos recém-nascidos das mulheres obesas com os das normais. Estes resultados constam da Tabela 11.

T A B E L A 11

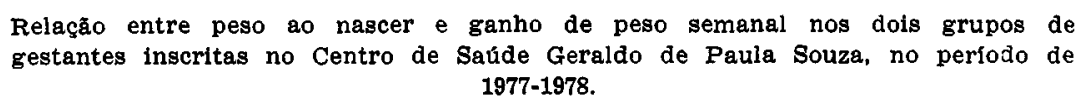

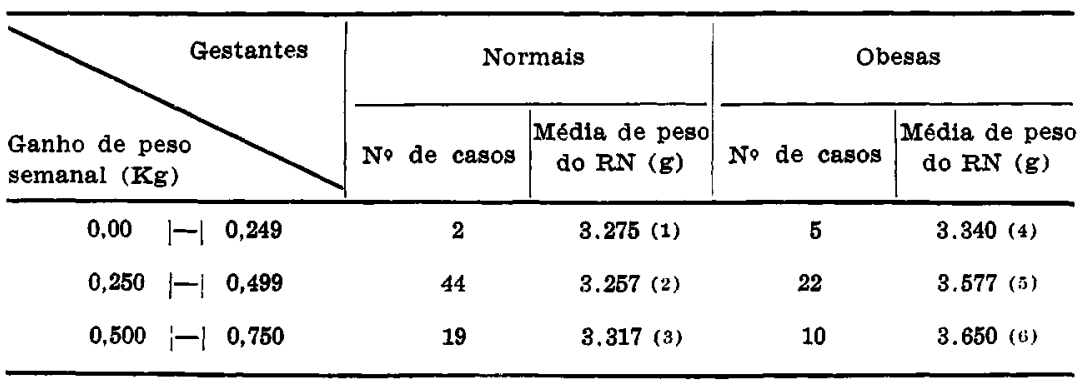

Houve diferença significativa ao nivel de $\mathrm{p}<0,05$ entre as médias de peso ao nascer dos ítens 2 e 5,3 e 6 . As demais não apresentaram diferença estatísticamente significativa.

Só foram estudados os recém-nascidos de 37 semanas e mais para evitar a interferência do haixo peso devido à prematuridade.

Apesar de não haver diferença significativa entre as médias de peso ao nascer, tanto no grupo das obesas como das normais, pode-se observar que quanto maior - ganho maior o peso do recém-nascido. Portanto, o ganho de peso é importante para o adequado crescimento intra-uterino.

\section{CONCLUSOES}

1. As variáveis idade, peso pré-gestacional e perímetro braquial mostraram que os dois grupos de gestantes apresentan características diferentes entre si.

2. As grávidas obesas apresentaram uma maior incidência de patologias, durante a gestação, que as normais, o que poderia indicar aumento do risco gravídico.

3. Os resultados aqui apresentados evidenciam o alto risco fetal a que os filhos das gestantes obesas estão expostos ou em relação a um elevado número de perdas fetais ou em razão da alta incidência de peso inadequado ao nascer $(<2.500 \mathrm{~g} \mathrm{e}$ $>4.000 \mathrm{~g}$ ) o que fica evidente pelo elevado coeficiente de mortalidade perinatal.

4. Constatou-se uma maior incidência de prematuridade no grupo das obesas que no das normais.

5. O peso dos conceptos das gestantes normais e obesas ao nascer são diferentes e suas distribuições também o são, não 
TANAKA, A.C. d'A. A importancia da associação obesidade e gravidez. Rev. Saúde públ., $\mathrm{s}$. Paulo, 15:291-307, 1981.

concorrendo para tal a paridade (que nas gestantes obesas é maior), a estatura, a idade e o perímetro braquial materno.

6. Tendo em vista o ganho de peso, durante a gravidez, existe prejuizo de crescimento fetal (menor peso ao nascer) quando a grávida ganha menos de $8 \mathrm{Kg}$ durante o período gestacional.

7. O peso pré-gestacional parece ser uma variável muito importante como meio de prognóstico de crescimento e desenvolvimento intra-uterino.

TANAKA, A.C. d'A. [The importance of the association of obesity and pregnancy]. Rev. Saúde públ., S. Paulo, 15:291-307, 1981.

ABSTRACT: Characteristics of the evolution of pregnancy in obese women were studied for their effect on newborn infants. Two control groups were observed - one of normal weight pregnant women, one of obese. The variables selected were: the socio-economic status of the family and the mother's age, height, arm circunference, prepregancy weight, total number of pregnancies, parity, weight gain during pregnancy, obstetric complications, birth weight, and fetal vitality. Results showed that pregnancy in obese women differs from that in normal weight women and that they show a larger incidence of obstetric complications. Children of obese mothers had a higher mortality rate principally in the perinatal period; moreover, there was also a higher rate of prematurity and a higher proportion of overweight babies among obese mothers. As a result, the distribution of the curve of the birth weight of infants of obese morthers was higher than that of infants of normal weight mothers. The conclusion reached was that whenever a pregnant obese woman reduced foot intake, with resultant insufficient weight gain, intrauterine growth was affected. Thus, it follows that pregnancy is not the best time for the obese mother to lose weight; for this reason, it is important that she receive adequate guidance in regard to diet. Obesity, therefore, is a factor contributing to high-risk pregnancy which can affect both mother and child.

UNITERMS: Obesity. Pregnancy. Birth weight. Pregnancy risk.

\section{REFERENCIAS BIBLIOGRAFICAS}

1. ALBRINK, M.J. Obesidad. In: Beenson, P.B. \& McDermott, W. Tratado de medioina interna de Ceoil Loeb. $12 \%$ ed. México, Ed. Interamericana, 1967. p. 1204-15.

2. ALMEIDA, P.A.M. de et al. Identificação e avaliaça dos fatores clínicos da gestação de alto risco. Rev. Saude putbl. S. Paulo, 9:417-25, 1975.

3. ALVARENGA, A.T. de et al. Indice de status sócio-econômico da familia de mulher grávida que frequlenta o Centro de Saúde Geraldo de Paula Souza da Faculdade de Saúde Pública da USP. Rev. Saride puibl,, S. Paulo, 7:351-67. 1973.
4. ALVAREZ-LAJONCHERE, C. Investigacion perinatal, Cuba, 1973. Rev. cub. Adm. Salud, 2:269-75, 1976.

5. BATISTA, N.A. Contribuigao para o estudo do recem-nascido de termo grande para $a$ idade gestacional. São Paulo, 1974. [Dissertação de Mestrado - Faculdade de Medicina da USP]

6. BRENNAN, R.E. Assessment of maternal nutrition. J. Amer, diet. Ass., $75: 152-4$, 1979.

7. BUTLER, N.R. \& BONHAM, D.G. Perinatal Mortality: the first report of the 1958 British Perinatal Mortality Survey. Edinburg. Livingstone, 1963. 
TANAKA, A.C. d'A. A importancia da associaçăo obesidade e gravidez. Rev. Saúde públ., $\mathbf{S}$. Paulo, 15:291-307, 1981.

8. CASTILHO, E.A. et al. Altura materna e peso da criança ao nascer. Rev. Saúde públ., S. Paulo, 10:233-7, 1976.

9. CHAMBERLAIN, R. et al. British births 1970. London, William Heinemann $\mathrm{Me}$ dical Books, 1975. p. 235-53.

10. CORMILLOT, A.F.J. et al. Obesidad. Buenos Aires, Ed. Med. Panamericana, 1977. p. 258-64.

11. CRAIG, L. Anthropometric determinants of obesity. In: Wilson. N. L., ed. Obesity. Philadelphia, F.A. Davis 1969. p. 13-23.

12. DELASCIO, D. \& ALMEIDA, P.A.M. de Propedeutica da gestagão de alto risco. Såo Paulo, Livraria Manolé, 1974. p. 67.

13. EDITORIAL. J. Amer, med. Ass., 211:492-3, 1970.

14. EDWARDS. I.E. et al. Pregnancy in the massively obese: course, outcome, and obesity prognosis of the infant. Amer. $J$. Obstet. Gynec., 131:479-83, 1978.

15. FISHER, J.J. \& FREY, I. Pregnancy and parturition in the obese patient. Obstet. and Gynec., 11:92-4, 1958.

16. KAWAKAMI, $S$. et al. Alteration of maternal body weight in pregnancy and postpartum. Keio J. Med., 26:58-62, 1977.

17. KIELL, N. Introduction. In: Kiell, N., ed. The psychology of obesity. Springfield, Ill., Charles C. Thomas, 1973. p. 9-15.

18. LAURENTI, R. et al. Mortalldade perinatal em São Paulo, Brasil. Rev. Saúde públ., S. Paulo, 9:115-24, 1975.

19. MAHAN, C.S. Revolution in obstetrics: pregnancy nutrition. J. Fla med. Ass., 66:367-72, 1979.

20. MATTHEWS, H.B. \& BRUCKE, M.G. der Normal expectancy in the extremely obese pregnant woman. J. Amer. med. Ass., 110:554_8, 1938.

21. NYIRJESY, $I$. et al. Clinical significance of total weight gain in pregnancy. I Primipara. Obstet. and Gynec., 32:391-6, 1968.

22. ODELL, L.L.D. MENGERT, W. F. The overweight obstetric patient. $J$. Amer. med. Ass., 128:87-90, 1945.

23. PECKHAM, C.H. \& CHRISTIANSON, R.E. The relationship between prepregnancy weight and certain obstetric factors. Amer. J. Obstet. Gynec., 111:1-7, 1971.

24. PETRY, J.A. Obesity with pregnancy. Obstet. and Gynec., $\mathrm{\tau}: 299-303,1956$.

25. ROBSON, J.R.K. Obesity; an overview. Med. Tms, New York, 107:49-50, 1979.
26. RUSH, P. et al. Antecedents of low birthweight in Hallen. New York City. Int. J. Epidem., 1:375-87, 1972.

27. SEMINARIO SOBRE LA PREVENCION DE LA MORBIDAD Y DE LA MORTALIDAD PERINATALES, Tours, 1969. Informe. Ginebra, Organizacion Mundial de la Salud, 1972. (OMS-Cuadernos de salud publica, 42).

28. SIMPSON, J.W. et al. Responsability of the obstetrician to the fetus. II. Influence of prepregnancy weight and pregnancy weight gain on birthweight. Obstet. and Gynec., 45:481-7, 1975.

29. SIQUEIRA, A.A.F. de Estudo de um conjunto de curvas antropométricas no diagnóstico de estado nutricional de gestantes e sua relaça com o tamanho do recém-nascido. São Pa:-lo, 1979. [Tese de Doutoramento - Faculdade de Saúde Pública da USP]

30. SIQUEIRA, A.A.F. de et al. A utllização de uma curva ponderal de gestantes normais no diagnóstico da desnutrição intra-uterina. Rev. Saúde públ., S. Paulo, 9:495-506, 1975.

31. SPIEGEL, M.R. Estatística. São Paulo, Mc Graw-Hill do Brasil, 1976.

32. SPRINGER, N.S. et al. Nutritional indexes of clients in a maternity and infant care project. I the target population. J. Amer. diet. Ass., 71:613-6, 1977.

33. TOMPKINS, W.T. \& WIEHL, D.G. Effect of nutrient suplements on obese patients during pregnancy. Obstet. and Gynec., $4: 365-73$, 1954.

34. TRACY, T.A. \& MILLER, G.L. Obstetric problems of the massively obese. Obstet. and Gynec., 33:204-8, 1969.

35. UDALL, J.N, et al. Interaction of materna] and neonatal obesity. Pediatrics, 62:17-21, 1978.

36. WEISS, W. \& JACKSON, E.L. Factores maternos que afectan el peso al nacer. In: Organizacion Panamericana de la Salud. Factores perinatales que afectan el desarrollo humano. Washington, D.C. 1972. p. 54-8. (OPAS-Publ. cient. 185).

37. WHITELAW, G.L. Influence of maternal obesity on subcutaneous fat in the newborn. Brit. med. J., 1:985-6, 1976.

38. WILSON, N.L. et al, The development and perpectuation of obesity: an overview. In: Wilson, N.L., ed. Obesity. Philadelphia, F.A. Davis, 1969. p. 3-12.

Recebido para publicacão em 05/12/1980 Aprovado para publicacáo em 11/03/1981 